\title{
Mandelstam-Leibbrandt prescription within path integral formulation
}

\section{Jerzy Przeszowski*}

University of Biatystok, Poland

E-mail: jprzeszo@alpha.uwb.edu.pl

\begin{abstract}
The path integral quantization for the Abelian gauge field $A_{\mu}$ in the null gauge condition: $n \cdot A=0$ with $n^{2}=0$, is presented. The equal-time (ET) and the light-front (LF) formulations, when $x^{0}$ and $x^{+}=\left(x^{0}+x^{3}\right) / \sqrt{2}$ are taken respectively as temporal evolution parameters, are compared. The Mandelstam-Leibbrandt (ML) prescription for non-covariant poles appears, when Wick's rotation is used for the ET path integrals. The LF path integrals need a modification of the Hamiltonian density before introducing Wick's rotation.
\end{abstract}

LIGHT CONE 2008 Relativistic Nuclear and Particle Physics

July 7-11 2008

Mulhouse, France

${ }^{*}$ Speaker. 


\section{Path integral for scalar field}

In the equal-time (ET) path integral quantization of a free scalar field $\phi$ one defines the generating functional for all Green functions as the phase-space path integral

$$
\begin{aligned}
Z[s] & \propto \int \mathscr{D} \phi \mathscr{D} \pi \exp i \int d^{4} x\left[\pi \partial_{0} \phi-\mathscr{H}_{c a n}\right] \times \exp i \int d^{4} x s \phi= \\
& \propto \int \mathscr{D} \phi \mathscr{D} \pi \exp i \int d^{4} x\left\{\pi \partial_{0} \phi-\frac{1}{2}\left[\pi^{2}+m^{2} \phi^{2}+(\vec{\nabla} \phi)^{2}\right]\right\} \times \exp i \int d^{4} x s \phi .
\end{aligned}
$$

In a practical calculation one uses the fundamental path integral Gaussian formula

$$
\begin{array}{r}
\int \mathscr{D} \phi \exp \left(-\frac{1}{2} \int d^{4} x \int d^{4} y \phi(y) A(y, x) \phi(x)+\int d^{4} x \phi(x) K(x)\right)= \\
=\exp \left\{\frac{1}{2} \iint d^{4} x d^{4} y K(x) A^{-1}(x, y) K(y)\right\} \times \exp -\frac{1}{2} \operatorname{Tr} \ln \mathbf{A}
\end{array}
$$

thus if one has a local differential operator $A(y, x)=\widehat{O} \delta^{4}(x-y)$ then one needs to know the inverse integral operator $A^{-1}(x, y)$. The path integrals in (1.1) can be transformed into a Gaussian form if one analytically continues to the Euclidean space by the Wick rotation

$$
x^{0} \mapsto \tau e^{-i \theta}, \quad \text { with } \quad 0 \leq \theta \leq \pi / 2
$$

and then integrates over the canonical momentum path-integral variable $\pi$

$$
Z[s] \propto \int \mathscr{D} \phi \exp -\frac{1}{2} \int d^{4} x_{E}\left\{\left(\partial_{\tau} \phi\right)^{2}+m^{2} \phi^{2}+(\vec{\nabla} \phi)^{2}\right\} \exp +\int d^{4} x_{E} s \phi,
$$

where $d^{4} x_{E}=d^{3} \vec{x} d \tau$. At last one may uniquely integrate over $\phi$ and then analytically continue back to the Minkowski space-time, which gives the final expression

$$
Z[s] \propto \exp \frac{i}{2} \iint d^{4} x d^{4} y s(x)\left[\square+m^{2}-i \varepsilon\right]^{-1}(x-y) s(y)
$$

where the Feynman propagator (with $\varepsilon>0$ )

$$
\left[\square+m^{2}-i \varepsilon\right]^{-1}(x)=\int \frac{d^{4} k}{(2 \pi)^{4}} \frac{e^{-i k \cdot x}}{-k_{0}^{2}+\vec{k}^{2}+m^{2}-i \varepsilon}
$$

appears naturally. The same causal prescription for poles in the Feynman propagator can be obtained without Wick's rotations by adding the damping term to the path integral over $\phi$

$$
Z[s] \propto \int \mathscr{D} \phi \exp \frac{i}{2} \int d^{4} x\left\{\left(\partial_{0} \phi\right)^{2}-m^{2} \phi^{2}-(\vec{\nabla} \phi)^{2}+i \varepsilon \phi^{2}\right\} \times \exp i \int d^{4} x s \phi .
$$

Thus for this academic case of path integral one may either use the Wick rotations or add the damping term - both lead to the causal propagator which agrees with the canonical quantization procedure. 


\section{ET formulation for the Abelian LC gauge}

In the standard formulation of a path integral for a gauge field theory one uses the BRST supersymmetry for establishing the proper form of effective action and functional integration variables (for example see [1]). Alternatively one may impose a gauge field condition as a classical constraint and reduce the system into its independent degrees of freedom, which then lead to a path integral over the effective phase space [2]. Thus if the gauge vector field component $A_{0}$, which due to the gauge symmetry has no canonical momentum, is removed by a gauge fixing condition, then one may work with a reduced unconstrained system.

Within the ET formulation one may equivalently choose either $A_{0}-A_{3}=0$ or $A_{0}+A_{3}=0$ as the LC gauge fixing condition for the Abelian gauge field theory. For fixing our attention let us take the first possibility and introduce $A_{0}=A_{3}$ into the Lagrangian density

$$
\begin{aligned}
\mathscr{L}_{L C} & =\frac{1}{2}\left(\partial_{0} A_{i}-\partial_{i} A_{3}\right)^{2}+\frac{1}{2}\left(\partial_{0} A_{3}-\partial_{3} A_{3}\right)^{2}-\frac{1}{4}\left(\partial_{i} A_{j}-\partial_{j} A_{i}\right)^{2}-\frac{1}{2}\left(\partial_{3} A_{i}-\partial_{i} A_{3}\right)^{2} \\
& +A_{3}\left(J^{0}+J^{3}\right)+A_{i} J^{i}, \quad i=1,2
\end{aligned}
$$

Then one easily finds that all canonical momenta are independent degrees of freedom

$$
\pi^{i}:=\frac{\partial \mathscr{L}_{L C}}{\partial\left(\partial_{0} A_{i}\right)}=\partial_{0} A_{i}-\partial_{i} A_{3}, \quad \pi^{3}:=\frac{\partial \mathscr{L}_{L C}}{\partial\left(\partial_{0} A_{3}\right)}=\partial_{0} A_{3}-\partial_{3} A_{3}
$$

which lead to the nonvanishing ET canonical Poisson brackets at equal $x^{0}$

$$
\left\{\pi^{i}\left(x^{0}, \vec{x}\right), A_{j}\left(x^{0}, \vec{y}\right)\right\}_{P B}=-\delta_{i j} \delta^{3}(\vec{x}-\vec{y}), \quad\left\{\pi^{3}\left(x^{0}, \vec{x}\right), A_{3}\left(x^{0}, \vec{y}\right)\right\}_{P B}=-\delta^{3}(\vec{x}-\vec{y}) .
$$

One may notice that the canonical Hamiltonian density, which is defined as

$$
\begin{aligned}
\mathscr{H}_{L C}:= & \pi^{i} \partial_{0} A_{i}+\pi^{3} \partial_{0} A_{3}-\mathscr{L}_{L C}=\frac{1}{2}\left(\pi^{i}\right)^{2}+\frac{1}{2}\left(\pi^{3}\right)^{2}+\pi^{i} \partial_{i} A_{3}+\pi^{3} \partial_{3} A_{3} \\
& +\frac{1}{4}\left(\partial_{i} A_{j}-\partial_{j} A_{i}\right)^{2}+\frac{1}{2}\left(\partial_{3} A_{i}-\partial_{i} A_{3}\right)^{2}-A_{3}\left(J^{0}+J^{3}\right)-A_{i} J^{i} .
\end{aligned}
$$

is not positively definite even for a free field case, when $J^{\mu}=0$.

As presented above, the canonical field variables form an unconstrained system, thus one may define the generating functional for the LC gauge as the path integral over the unconstrained phase space

$$
Z_{L C}\left[J^{\mu}\right] \propto \int \mathscr{D} A_{i} \mathscr{D} A_{3} \mathscr{D} \pi^{i} \mathscr{D} \pi^{3} \exp i \int d^{4} x\left[\pi^{i} \partial_{0} A_{i}+\pi^{3} \partial_{0} A_{3}-\mathscr{H}_{L C}\right]
$$

and then integrate over the momentum variables $\pi^{i}$ and $\pi^{3}$

$$
\begin{aligned}
\int \mathscr{D} \pi^{i} \mathscr{D} \pi^{3} \exp i \int d^{4} x\left[\pi^{i} \partial_{0} A_{i}+\pi^{3} \partial_{0} A_{3}-\frac{1}{2}\left(\pi^{i}\right)^{2}-\frac{1}{2}\left(\pi^{3}\right)^{2}-\pi^{i} \partial_{i} A_{3}-\pi^{3} \partial_{3} A_{3}\right] \\
\propto \exp \frac{i}{2} \int d^{4} x\left[\left(\partial_{0} A_{3}-\partial_{3} A_{3}\right)^{2}+\left(\partial_{0} A_{i}-\partial_{i} A_{3}\right)^{2}\right]
\end{aligned}
$$


Thus effectively one obtains the path integral over the independent gauge fields $A_{i}$ and $A_{3}$ for the LC gauge condition

$$
\begin{aligned}
Z_{L C}\left[J^{\mu}\right] \propto & \int \mathscr{D} A_{i} \mathscr{D} A_{3} \exp i \int d^{4} x\left[A_{3}\left(J^{0}+J^{3}\right)+A_{i} J^{i}\right] \times \\
& \times \exp \frac{i}{2} \int d^{4} x\left[\left(\partial_{0} A_{3}-\partial_{3} A_{3}\right)^{2}+\left(\partial_{0} A_{i}-\partial_{i} A_{3}\right)^{2}\right] \times \\
& \times \exp -\frac{i}{2} \int d^{4} x\left[\frac{1}{2}\left(\partial_{i} A_{j}-\partial_{j} A_{i}\right)^{2}+\left(\partial_{3} A_{i}-\partial_{i} A_{3}\right)^{2}\right],
\end{aligned}
$$

where remaining integrals contain partial differential operators, which have to be inverted. Just like in Eq.(1.3) one may introduce the ET Wick rotation, which leads to the Euclidean space, where the inverse differential operators are unique and then one continues back to the Minkowski space-time. One finds that this prescription for the path integral over $A_{3}$ can be effectively expressed as the following modification

$$
\begin{gathered}
\left.Z_{1}\left[J^{\mu}\right] \propto \int \mathscr{D} A_{3} \exp i \int d^{4} x A_{3}\left[J^{0}+J^{3}+\left(\partial_{0}-\partial_{3}+i \varepsilon \partial_{3}\right)\right) \partial_{i} A_{i}\right] \times \\
\times \exp -\frac{i}{2} \int d^{4} x A_{3}\left[\left(\partial_{0}-\partial_{3}+i \varepsilon \partial_{3}\right)^{2}\right] A_{3},
\end{gathered}
$$

where $\varepsilon>0$ is an infinitesimal parameter. Contrary to the scalar field case a different result will follow if one introduces the damping term $i \varepsilon A_{3}^{2}$

$$
\begin{gathered}
\left.Z_{1}\left[J^{\mu}\right] \propto \int \mathscr{D} A_{3} \exp i \int d^{4} x A_{3}\left[J^{0}+J^{3}+\left(\partial_{0}-\partial_{3} \partial_{3}\right)\right) \partial_{i} A_{i}\right] \times \\
\times \exp -\frac{i}{2} \int d^{4} x A_{3}\left[\left(\partial_{0}-\partial_{3}\right)^{2}-i \varepsilon\right] A_{3},
\end{gathered}
$$

thus these two prescriptions are no longer equivalent. The remaining path integral over $A_{i}$ by the Wick rotation prescription becomes modified as follows

$$
\begin{aligned}
Z_{2}\left[J^{\mu}\right] & \propto \int \mathscr{D} A_{i} \exp -\frac{i}{2} \int d^{4} x A_{i}\left[\partial_{0}^{2}-\partial_{3}^{2}-\Delta_{\perp}-i \varepsilon\right] A_{i} \\
& \times \exp i \int d^{4} x A_{i}\left[J^{i}+\partial_{i} \frac{1}{\partial_{0}-\partial_{3}+i \varepsilon \partial_{3}}\left(J^{0}+J^{3}\right)\right] .
\end{aligned}
$$

Accordingly by introducing the ET Wick rotation one may effectively regularize all inverse differential operators and finally one obtains the gauge field propagator in the LC gauge as the welldefined distribution in the momentum space

$$
\mathscr{D}_{\mu \nu}^{L C}(k)=\left[g_{\mu \nu}-\frac{n_{\mu} k_{v}+n_{v} k_{\mu}}{[n \cdot k]}\right] \frac{1}{k^{2}+i \varepsilon}, \quad \frac{1}{[n \cdot k]}=\frac{1}{k_{0}-k_{3}+i \varepsilon^{\prime} k_{3}},
$$

where $n_{0}=n_{3}=1, n_{i}=0$. Evidently the above prescription for the noncovariant pole is equivalent to the well-known ML prescription [3], [4]

$$
\left[\frac{1}{n \cdot k}\right]_{M L}=\frac{1}{k_{0}-k_{3}+i \varepsilon \operatorname{sgn}\left(k_{0}+k_{3}\right)}=\frac{k_{0}+k_{3}}{\left(k_{0}-k_{3}\right)\left(k_{0}+k_{3}\right)+i \varepsilon^{\prime}}
$$

and the above path integral formulation agrees with the canonical quantization procedure [5]. 


\section{LF Weyl (LFW) gauge}

In the LF formulation one usually takes $x^{+}$as the temporal evolution parameter, where for any 4-vector $a^{\mu}: a^{+}=\left(a^{0}+a^{3}\right) / \sqrt{2}$ and $a^{-}=\left(a^{0}-a^{3}\right) / \sqrt{2}$. Thus one has two null axial gauge conditions: the light-cone (LC) gauge $A_{-}=A^{+}=0$ and the LF Weyl (LFW) gauge $A_{+}=A^{-}=0$. The LF canonical quantization procedure shows that these two gauges are essentially different and the ML prescription for non-covariant poles arises only for the LFW gauge [6]. In the LF formulation, due to gauge symmetry, the gauge field component $A_{+}$has no canonical momentum, thus one may impose the LFW gauge fixing condition $A_{+}=0$ directly into the Lagrangian density

$$
\mathscr{L}_{L F W}=\frac{1}{2}\left(\partial_{+} A_{-}\right)^{2}+\partial_{+} A_{i}\left(\partial_{-} A_{i}-\partial_{i} A_{-}\right)-\frac{1}{4}\left(\partial_{i} A_{j}-\partial_{j} A_{i}\right)^{2}+\mathbf{A}_{\mu} \mathbf{J}^{\mu}
$$

where for any 4-vector $\mathbf{a}^{\mu}=\left(a^{+}=0, a^{-}, a^{i}\right)$. The LF canonical momenta are defined as follows

$$
\pi^{i}=\frac{\partial \mathscr{L}_{L C W}}{\partial\left(\partial_{+} A_{i}\right)}=\partial_{-} A_{i}-\partial_{i} A_{-}, \quad \pi^{-}=\frac{\partial \mathscr{L}_{L C W}}{\partial\left(\partial_{+} A_{-}\right)}=\partial_{+} A_{-},
$$

which means that there are primary constraints: $\chi^{i}=\pi^{i}-\partial_{-} A_{i}+\partial_{i} A_{-} \simeq 0$. Further canonical analysis shows that these constraints are second class and the independent nonvanishing LF Poisson brackets at equal $x^{+}$are

$$
2\left\{A_{i}\left(x^{+}, \bar{x}\right), \partial_{-} A_{j}\left(x^{+}, \bar{y}\right)\right\}_{P B}=\delta_{i j} \delta^{3}(\bar{x}-\bar{y}), \quad\left\{\left(\pi^{-}-\partial_{i} A_{i}\right)\left(x^{+}, \bar{x}\right), A_{-}\left(x^{+}, \bar{y}\right)\right\}_{P B}=-\delta^{3}(\bar{x}-\bar{y}) .
$$

The canonical Hamiltonian density can be evaluated as follows

$$
\mathscr{H}_{L F W}:=T^{+-}=\pi^{i} \partial_{+} A_{i}+\pi^{-} \partial_{+} A_{-}-\mathscr{L}_{L F W}=\frac{1}{2}\left(\pi^{-}\right)^{2}+\frac{1}{4}\left(\partial_{i} A_{j}-\partial_{j} A_{i}\right)^{2}-\mathbf{A}_{\mu} \mathbf{J}^{\mu}
$$

where $\pi^{i}$ momenta are eliminated from the constraint relation $\chi^{i}=0$ and evidently it is positively definite for $J^{\mu}=0$.

Since the system has second class constraints, then the generating functional is defined as the path integral over the phase space variables [7]

$$
\begin{gathered}
Z_{L F W}\left[\bar{J}^{\mu}\right] \propto \int \mathscr{D} A_{i} \mathscr{D} A_{-} \mathscr{D} \pi^{i} \mathscr{D} \pi^{-} \delta\left[\pi^{i}-\partial_{-} A_{i}+\partial_{i} A_{-}\right] \exp i \int d^{4} x\left[\pi^{i} \partial_{+} A_{i}+\pi^{-} \partial_{+} A_{-}\right] \times \\
\times \exp -i \int d^{4} x\left[\frac{1}{2}\left(\pi^{-}\right)^{2}+\frac{1}{4}\left(\partial_{i} A_{j}-\partial_{j} A_{i}\right)^{2}\right] \exp i \int d^{4} x \mathbf{A}_{\mu} \mathbf{J}^{\mu}
\end{gathered}
$$

where the integration over the dependent momenta $\pi^{i}$ can be trivially done

$$
\begin{array}{rl}
Z\left[\bar{J}^{\mu}\right] \propto \int \mathscr{D} A_{i} & \mathscr{D} A_{-} \mathscr{D} \pi^{-} \exp i \int d^{4} x\left[\partial_{+} A_{i}\left(\partial_{-} A_{i}-\partial_{i} A_{-}\right)+\pi^{-} \partial_{+} A_{-}\right] \times \\
\times & \exp -i \int d^{4} x\left[\frac{1}{2}\left(\pi^{-}\right)^{2}+\frac{1}{4}\left(\partial_{i} A_{j}-\partial_{j} A_{i}\right)^{2}\right] \exp i \int d^{4} x \mathbf{A}_{\mu} \mathbf{J}^{\mu} .
\end{array}
$$

Next the integral over momentum $\pi^{-}$is similar to Eq.(2.6), thus effectively one derives the path integral over the independent gauge field components $A_{i}$ and $A_{-}$

$$
\begin{aligned}
Z\left[J^{\mu}\right] \propto & \int \mathscr{D} A_{i} \mathscr{D} A_{-} \exp i \int d^{4} x\left[\partial_{+} A_{i}\left(\partial_{-} A_{i}-\partial_{i} A_{-}\right)\right] \times \\
& \times \exp i \int d^{4} x\left[\frac{1}{2}\left(\partial_{+} A_{-}\right)^{2}-\frac{1}{4}\left(\partial_{i} A_{j}-\partial_{j} A_{i}\right)^{2}\right] \exp i \int d^{4} x \mathbf{A}_{\mu} \mathbf{J}^{\mu}
\end{aligned}
$$


Before integrating over $A_{i}$ one may introduce the LF Wick rotation: $x^{+} \mapsto \tau e^{-i \theta}$ with $0 \leq \theta \leq \pi / 2$, which effectively leads to the modified path integral (with $\varepsilon>0$ )

$$
\begin{aligned}
Z\left[J^{\mu}\right] \propto & \int \mathscr{D} A_{i} \mathscr{D} A_{-} \exp i \int d^{4} x\left[\partial_{+} A_{i}\left(\partial_{-} A_{i}-\partial_{i} A_{-}\right)+i \varepsilon A_{i}^{2}\right] \times \\
& \times \exp i \int d^{4} x\left[\frac{1}{2}\left(\partial_{+} A_{-}\right)^{2}-\frac{1}{4}\left(\partial_{i} A_{j}-\partial_{j} A_{i}\right)^{2}+\mathbf{A}_{\mu} \mathbf{J}^{\mu}\right] .
\end{aligned}
$$

However this means that the LF Wick rotation leaves $\left[\partial_{+}^{2}\right]^{-1}$ without any regularization and unfortunately the gauge field propagator stays ill-defined in the path integral formulation. The problem arises since the noncovariant pole at $k_{+}=0$ is not avoided by the LF Wick rotation, contrary to the covariant pole at $k_{+}=k_{\perp}^{2} / 2 k_{-}$, which obtains the standard $i \varepsilon$ prescription. This suggests the possible solution of the problem - one should push the position of noncovariant pole staying within the LFW gauge. This seems to be self-contradictory within the standard path integral formulation, thus one may resort to its modification

$$
\begin{aligned}
Z_{\alpha}\left[\bar{J}^{\mu}\right] \propto & \int \mathscr{D} A_{i} \mathscr{D} A_{-} \mathscr{D} \pi^{i} \mathscr{D} \pi^{-} \delta\left[\pi^{i}-\partial_{-} A_{i}+\partial_{i} A_{-}\right] \times \\
& \times \exp i \int d^{4} x\left[\pi^{i} \partial_{+} A_{i}+\pi^{-} \partial_{+} A_{-}-\mathscr{H}_{\alpha}\right]
\end{aligned}
$$

with the new Hamiltonian density $\mathscr{H}_{\alpha}=T^{+-}+\alpha T^{++}$, where $\alpha>0$ is a small regularization parameter, which should be removed $\alpha \rightarrow 0$ only for the final expressions. One may notice that this modified Hamiltonian density $\mathscr{H}_{\alpha}$ is not positively definite, since the Lagrangian density (3.1) gives the energy-momentum tensor component

$$
T^{++}=\pi^{-} \partial_{-} A_{-}+\left(\partial_{-} A_{i}\right)^{2}-\partial_{i} A_{-} \partial_{-} A_{i}
$$

When one integrates over $\pi^{i}$ in (3.10), then one finds the unconstrained modified phase space integral, which is written explicitly as follows

$$
\begin{aligned}
Z_{\alpha}\left[\bar{J}^{\mu}\right] & \propto \int \mathscr{D} A_{i} \mathscr{D} A_{-} \mathscr{D} \pi^{-} \exp i \int d^{4} x\left[\partial_{+} A_{i}\left(\partial_{-} A_{i}-\partial_{i} A_{-}\right)+\pi^{-} \partial_{+} A_{-}\right] \times \\
& \times \exp -i \alpha \int d^{4} x\left[\pi^{-} \partial_{-} A_{-}+\left(\partial_{-} A_{i}\right)^{2}-\partial_{i} A_{-} \partial_{-} A_{i}\right] \times \\
& \times \exp -i \int\left[\frac{1}{2}\left(\pi^{-}\right)^{2}+\frac{1}{4}\left(\partial_{i} A_{j}-\partial_{j} A_{i}\right)^{2}\right] \exp i \int d^{4} x \mathbf{A}_{\mu} \mathbf{J}^{\mu}
\end{aligned}
$$

and further integration over momentum $\pi^{-}$leads to the path integral over the independent gauge fields $A_{i}$ and $A_{-}$

$$
\begin{aligned}
Z_{\alpha}\left[J^{\mu}\right] \propto & \int \mathscr{D} A_{i} \mathscr{D} A_{-} \exp i \int d^{4} x\left[\partial_{+} A_{i}\left(\partial_{-} A_{i}-\partial_{i} A_{-}\right)\right] \times \\
& \times \exp i \int d^{4} x\left[\frac{1}{2}\left[\left(\partial_{+}-\alpha \partial_{-}\right) A_{-}\right]^{2}-\frac{1}{4}\left(\partial_{i} A_{j}-\partial_{j} A_{i}\right)^{2}\right] \times \\
& \times \exp -i \alpha \int d^{4} x\left[\left(\partial_{-} A_{i}\right)^{2}-\partial_{i} A_{-} \partial_{-} A_{i}\right] \exp i \int d^{4} x \mathbf{A}_{\mu} \mathbf{J}^{\mu}
\end{aligned}
$$


This last path integral can be rewritten as

$$
\begin{aligned}
Z_{\alpha}\left[J^{\mu}\right] \propto & \int \mathscr{D} A_{i} \mathscr{D} A_{-} \exp i \int d^{4} x\left[\tilde{\partial}_{+} A_{i}\left(\partial_{-} A_{i}-\partial_{i} A_{-}\right)\right] \times \\
& \times \exp i \int d^{4} x\left[\frac{1}{2}\left(\tilde{\partial}_{+} A_{-}\right)^{2}-\frac{1}{4}\left(\partial_{i} A_{j}-\partial_{j} A_{i}\right)^{2}\right] \exp i \int d^{4} x \mathbf{A}_{\mu} \mathbf{J}^{\mu}
\end{aligned}
$$

where $\tilde{\partial}_{+}:=\partial_{+}-\alpha \partial_{-}$. Now the LF Wick rotation $x^{+} \mapsto \tau e^{-i \theta}$, with $0 \leq \theta \leq \pi / 2$, modifies the partial differential operator as

$$
\partial_{+}-\alpha \partial_{-} \longmapsto \partial_{+}-\alpha \partial_{-}+i \varepsilon \alpha \partial_{-}
$$

and finally the path integrals lead to modified gauge field propagator in the LFW gauge

$$
\mathscr{D}_{\mu v}^{L F W}(k)=\left[g_{\mu v}-\frac{n_{\mu}^{W} \tilde{k}_{v}+n_{v}^{W} \tilde{k}_{\mu}}{\left[n^{W} \cdot \tilde{k}\right]}\right] \frac{1}{\tilde{k}^{2}+i \varepsilon}, \quad \frac{1}{\left[n^{W} \cdot \tilde{k}\right]}=\frac{1}{k_{+}-\alpha k_{3}+i \varepsilon^{\prime} \alpha k_{3}}
$$

where $\tilde{k}_{+}=k_{+}-\alpha k_{-}, \tilde{k}_{-}=k_{-}, \tilde{k}_{i}=k_{i}$, and $n_{-}^{W}=1, n_{+}^{W}=n_{i}^{W}=0$. Evidently this propagator has a non-standard form, but nevertheless it can be used for the perturbative calculations of Feynman's diagrams. One may expect that when the limit $\alpha \rightarrow 0$ is taken after integrating over $k_{+}$, then one obtains the same results as in the ET approach with the ML prescription.

\section{Conclusions}

- within the ET path integral formulation Wick's rotation leads to the ML prescription for noncovariant poles contrary to the damping factor

- for $\alpha>0$ the LF Wick rotation leads to the causal prescription for noncovariant poles, which is similar to the ML prescription

- the causal prescriptions for noncovariant poles follow from Hamiltonians, which are not positive definite

\section{References}

[1] A. Bassetto, G. Nardelli and R. Soldati, Yang-Mills Theories in Algebraic Non-covariant Gauges, World Scientific Publishing, Singapore 1991.

[2] L. Faddeev and R. Jackiw, Phys.Rev.Lett. 60, 1692 (1988).

[3] S. Mandelstam, Nucl.Phys.B 213, 149 (1983).

[4] G. Leibbrandt, Phys.Rev.D 29, 1699 (1984).

[5] A. Bassetto, M. Dalbosco, I. Lazzizzera and R. Soldati, Phys.Rev.D 31, 2012 (1985).

[6] M. Morara and R. Soldati, Phys.Rev.D 58, 105011 (1998).

[7] P. Senjanovič, Ann.Phys 100, 227 (1976). 